\title{
Speed of apparent motion and the wagon-wheel effect
}

\author{
DAVID J. FINLAY \\ University of Newcastle, Newcastle, New South Wales, Australia \\ and \\ PETER C. DODWELL \\ Queen's University, Kingston, Ontario, Canada
}

\begin{abstract}
The speed of apparent movement of a stroboscopically illuminated spoked wheel was measured by counting the number of spokes passing a particular position per unit of time. This measure gave very stable values of speed, which compared closely to predicted speeds calculated from stimulus onset asynchrony and angular displacement values. The results provide, for the first time, a reliable measure of speed of one form of apparent motion. The traditional explanation for the wagon-wheel effect in terms of beta motion is discussed in light of several differences between the classical demonstrations of apparent motion and stroboscopic motion as seen on the wagon wheel.
\end{abstract}

Regularly repeating stimuli, such as the spokes of a rotating wheel, often appear to reverse their direction of movement when presented stroboscopically. This effect, known as the wagon-wheel effect (WWE), is commonly seen in motion pictures and serves to highlight the artificial nature of all movement perception experienced when watching a movie. When a clockwise rotating wheel is illuminated stroboscopically (e.g., by the frames of a film), the successive exposures will catch the spokes slightly before, after, or exactly in the position occupied by other spokes on previous exposures. The wheel will appear to rotate in a counterclockwise or clockwise direction or will appear to be stationary. Wertheimer $(1912 / 1961)$ proposed that the WWE is a form of beta motion, the form of apparent object motion seen between a pair of briefly presented stimuli under suitable spatial and temporal separations. More complex forms of apparent motion, such as that between two- or even threedimensional outlined geometric figures, have been studied extensively (e.g., Foster, 1975; Kolers, 1972), although, as we discuss later, it is not certain that they can all be classed as beta motion.

In a previous study, we examined the WWE under a variety of stimulus and exposure conditions (Finlay, Dodwell, \& Caelli, 1984) and argued that beta motion was an inadequate explanation for some of the effects we observed with even the simplest display, in this case a rotat-

This research was undertaken while the first author was on sabbatical leave at Queen's University. The work was supported by Grant A0044 from the Natural Sciences and Engineering Research Council of Canada to P. C. Dodwell and D. W. Muir. D. J. Finlay was supported by a special grant from Queen's University, and P. C. Dodwell by a Killam Research Fellowship. Address requests for reprints to P. C. Dodwell, Department of Psychology, Queen's University, Kingston, Ontario, Canada, K7L 3N6. ing black wheel with four white spokes illuminated stroboscopically. We found that nearest-neighbor relationships (NNRs) would predict motion velocity (direction and speed), although the specification of NNRs was not itself simple, requiring the postulation of a filter to mediate spatiotemporal separations. In other words, apparent motion (AM) could not be predicted from simple physical or geometric principles. Moreover, at moderate and high rates of flicker, both visual persistence and masking complicate the picture, and AM can be generated between spokes that are currently illuminated and persisting images of other (or the same) spokes. The actual rotation rate of the wheel can bias the direction of AM at certain flash frequencies. It was also necessary to postulate a topdown organizational process to determine a best fit of the complex spatiotemporal display to the smooth, uniform motion actually perceived. We were able to model the process with some success, but the picture that emerged was of a highly interactive system in which the WWE is strongly constrained by coding and organizing characteristics of the observer's visual system.

There have been many discussions about the perceived velocity of apparent motion, but no successful analysis of the situation has been given before in detail. Kolers (1972), for instance, argued that the calculation of perceived velocity was not possible because it was not clear what the appropriate temporal parameter was. This conclusion was reached after lengthy consideration of both interstimulus interval (ISI) and stimulus onset asynchrony (SOA) measures. A similar problem arises in deciding about the appropriate spatial parameter. For instance, many studies have shown that proximal distances may not be the appropriate measures of interstimulus separation (Attneave \& Block, 1973; Finlay, 1982; Rock \& Ebenholtz, 1962). Kaufman, Cyrulnik, Kaplowitz, Melnick, 
and Stoff (1971), with a very limited set of stimulus conditions, proposed that AM occurs at velocities greater than those at which objects in real motion can be seen clearly. Bartley (1941) expressed the belief that AM may have a narrower range of speeds than does real motion (RM). No one has previously found a paradigm within which speed of AM could be accurately and easily measured, so there has been no ready way to compare AM and RM.

The issues we address are (1) the nature of the spatial and temporal parameters underlying the AM seen in the WWE, (2) the question of whether the WWE is related to beta motion, and (3) the question of how perceptual organization enters into the picture.

\section{GENERAL METHOD}

\section{Subjects}

Complete data from one subject (D.F.) were collected in the three experiments reported. Less extensive results were obtained for other subjects in each of the experiments as a check on generality. The results of these subjects showed no differences from the highly regular pattern of results shown by D.F.

\section{Materials}

A direct-current synchronous motor (Bodine Electric Companies, Series 200 Control Motor NSH 1QR Type) with speed control was used to rotate a $60-\mathrm{mm}$ black disk with four equally spaced, $1 \mathrm{~mm}$ wide, white radii in a counterclockwise direction. At the viewing distance of $1,000 \mathrm{~mm}$, the black disk subtended a visual angle of $3.5^{\circ}$. The stroboscopic illumination was produced with a detachable light box and timing equipment from a Gerbrands four-field tachistoscope. This device allowed strobe on and off times (and hence strobe frequency), as well as the illuminance on the display, to be varied independently.

\section{EXPERIMENT 1: Apparent Motion as a Function of SOA}

\section{Procedure}

The speed of the wheel was constant at $10 \mathrm{~Hz}$ and the wheel rotated in a counterclockwise direction. We used temporal frequencies in the range of about $8-16 \mathrm{~Hz}$. Under these conditions the spokes appear to move at a steady angular velocity (for a given strobe frequency), which can be easily measured by counting the number of spokes appearing to pass a given position (usually the lower vertical radius) per unit of time. The display was always viewed binocularly.

Eleven "starting point" combinations of strobe on and strobe off durations were chosen: 40/60, 50/50,60/40,75/25, 25/75, $40 / 51,40 / 41,60 / 31,75 / 16,75 / 2$, and $1 / 99 \mathrm{msec}$, with the first number of each pair being the on duration and the second the off time, or ISI. For a given combination of duration and ISI selected from the above list, the apparent rotation speed was measured and the direction of movement noted. The ISI was then increased by $1 \mathrm{msec}$, thereby changing the effective strobe frequency, and measurements were taken of apparent speed and direction. The ISI was again increased by $1 \mathrm{msec}$ and further measurements were made. This process continued until the speed of AM was too fast to measure or until the AM display became unstable. Then, returning to the same starting point, the ISI was decreased in 1-msec steps until the same conditions of instability were met. Likewise, with the ISI constant, the duration value was increased in 1-msec steps from the starting point and measures of speed and direction were made for each millisecond change. The duration values were then decreased from the starting point. When a series of both increases and decreases to both ISI and duration had been completed for a given starting point, another starting point was selected and the process was repeated.

In this way about 10 data points were obtained for each approximately $0.2-\mathrm{Hz}$ increment in strobe frequency between 8 and $16 \mathrm{~Hz}$. As is apparent, the values selected for starting points provided a wide range of durations (from 1 to $74 \mathrm{msec}$ ) and ISI values (from 2 to $99 \mathrm{msec}$ ) and allowed for an examination of symmetry in the effects of duration and ISI. Some of the values chosen replicate those used in an earlier study (Finlay et al., 1984).

\section{Results}

In Figure 1, speed of rotation is plotted against strobe frequency, both measured in hertz. The apparent speed of the wheel increases linearly with respect to strobe frequency, regardless of the duration/ISI combination. That is, the speed is independent of the stimulus duration and the data are well described by an onset-to-onset measure, or SOA. This contrasts with normal beta motion between two discrete points, which cannot be fully described by SOA (Kolers, 1972); in that case the actual temporal parameter is difficult to specify. A second point is that, under our conditions, apparent speed is symmetrical for clockwise and counterclockwise motion; the actual direction of wheel rotation has no biasing effect for any of the stimulus duration values used. It is interesting to note that although SOA determines the speed of AM, the on component of the strobe cycle determines certain other features of the very complex displays that are generated. For instance, during a 1-msec exposure at the rotation rate used, a given spoke moves $3.6^{\circ}$, whereas during a 75msec exposure it travels $270^{\circ}$. At the 1 -msec exposure four thin white spokes are seen, whereas at longer durations wedges of various sizes appear, and they generally appear to be moving at a steady angular velocity. Obviously, with passes as great as $270^{\circ}$, the path swept by one spoke will overlap that swept by its predecessor, so doubly stimulated overlap regions are also seen, as are, in some displays, light and dark regions resembling Mach bands. Regardless of these large variations in display properties, the speed of apparent motion remains the same for a given SOA. Thus the perceived AM is not affected by the area swept by each spoke as it is illuminated. Apparently it is the physical leading edge of a wedge, created by the illuminated spoke, that determines the speed and direction of AM.

The direction of AM is readily understood. At $10 \mathrm{~Hz}$ (and at $13.3 \mathrm{~Hz}$ ), the four-spoke wheel exactly repeats itself and the wheel appears to be stationary. For strobe rates just above $10 \mathrm{~Hz}$ (and $13.3 \mathrm{~Hz}$ ), the spokes fall short of exact repetition and, as with classical AM, movement is from the initial to the subsequent exposure; that is, clockwise. The counterclockwise motion is similarly explained. For strobe rates of about 9 and $11 \mathrm{~Hz}$, the display becomes unstable, as the currently illuminated spoke finds itself approximately midway between two spoke positions exposed on the previous illuminations. Crossovers from clockwise to counterclockwise as a function of strobe 


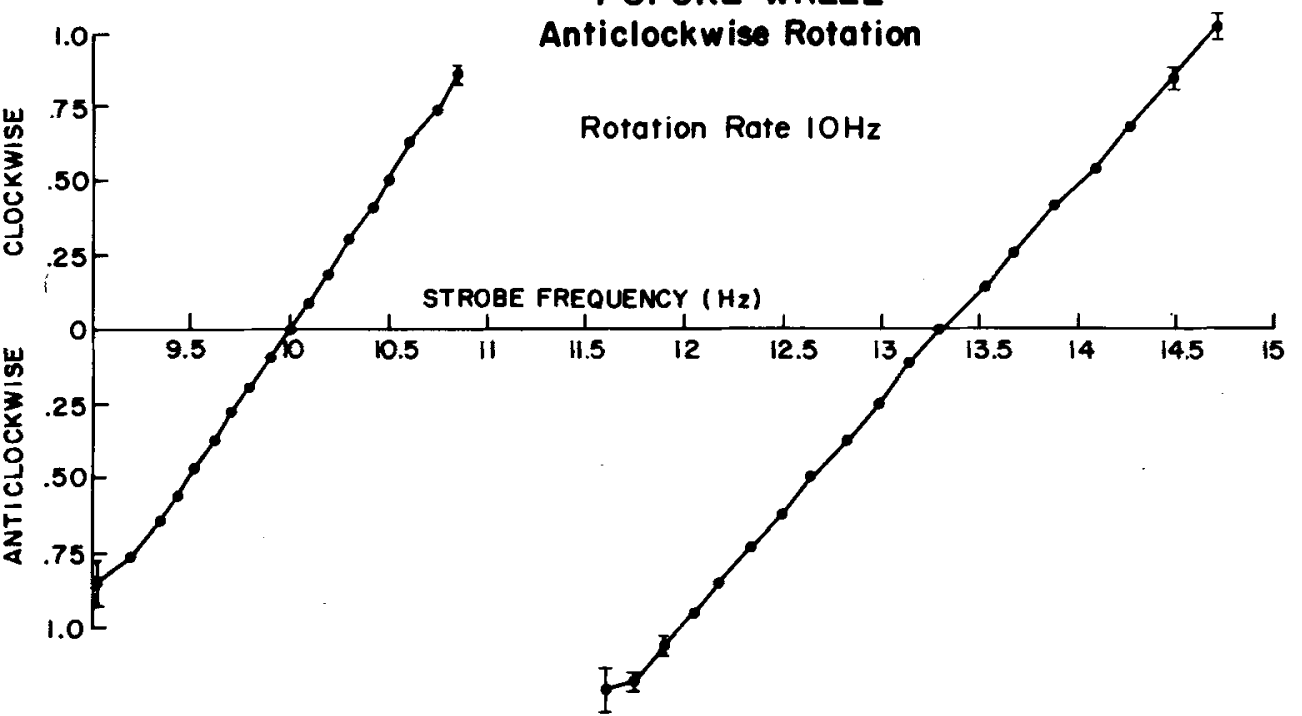

Figure 1. Averaged judged rotation speeds for all interstimulus interval combinations (strobe frequencies) and judged direction of movement. The error bars are standard errors, which for most points are included within the data point markers. The crossing points at 10 and $13.3 \mathrm{~Hz}$ are points of exact repetition for the pattern and rotation rate used.

rate are very rapid in these regions (cf. Finlay et al., 1984). Experiment 1 thus demonstrated that SOA provides an adequate description of the temporal parameter underlying the WWE and, furthermore, that the actual direction of the movement of the wheel did not bias the speed of apparent rotation.

\section{EXPERIMENT 2: Effect of Wheel Rotation Rate}

\section{Procedure}

Only one combination of duration and ISI, 1/99, was used as a starting point in this experiment. The ISI was varied in 1-msec steps, as before, to produce a range of temporal frequencies from 8 to $12.25 \mathrm{~Hz}$. The speed and direction of the AM were measured at each increment or decrement in ISI over the entire range of temporal frequencies. This was repeated for each of the following rotation speeds of the wheel: $1,2,2.2,3,4,5,6$, and $7 \mathrm{~Hz}$.

\section{Results}

The effects of changing strobe frequency for different rotation rates of the wheel are illustrated in Figure 2. Several of the curves, for example, those for 6- and 4- $\mathrm{Hz}$ rotation rates, have two limbs. It is not possible to connect them because AM becomes unstable in the regions between the limbs, for instance, between the 9 - and $10-\mathrm{Hz}$ strobe rates for the $6-\mathrm{Hz}$ rotation rate. Apart from these regions, the obvious feature of the curves is their extremely regular nature.

These data can be explained on exactly the same basis as the findings of Experiment 1. Consider the strobe frequency of $8 \mathrm{~Hz}$ (duration $1 \mathrm{msec}$, ISI $124 \mathrm{msec}$ ); at this strobe frequency the 2-, 4-, and 6- $\mathrm{Hz}$ rotation rates will produce exact repetitions by traveling $90^{\circ}, 180^{\circ}$, and $270^{\circ}$, respectively. Small increments or decrements from these values induce prograde or retrograde $\mathrm{AM}$, as before. Similarly, the 3-, 5-, and 7- $\mathrm{Hz}$ rotation rates cross the zero $A M$ axis at 12,10 , and $9.33 \mathrm{~Hz}$, respectively, the values at which the spokes travel through $270^{\circ}, 180^{\circ}$, and $90^{\circ}$. The three curves also converge in a point at $8 \mathrm{~Hz}$, with rapid counterclockwise AM. At that strobe rate, the successive displacements are $315^{\circ}, 225^{\circ}$, and $135^{\circ}$, respectively, that is, just halfway between exact repetitions. These are the greatest displacements possible and perceived speed becomes unstable, as does the direction of apparent rotation. Following similar logic, the other crossing points can be explained. For example, a rotation rate of $2.2 \mathrm{~Hz}$ was selected to include a noninteger value. At this rotation rate, the wheel traverses $0.792^{\circ} / \mathrm{msec}$ per flash and will therefore travel $90^{\circ}$ (an exact repetition rate) after approximately $113 \mathrm{msec}-\mathrm{a}$ strobe rate of $8.8 \mathrm{~Hz}$.

The data for the $1-\mathrm{Hz}$ rotation rate appear to provide a lower bound to the effect. As in the other curves, the missing data refer to points at which the AM was either unstable, that is, oscillating, or too fast to measure. It is important to note here, as we did earlier (Finlay et al., 1984), that the apparent number of spokes seen can increase with strobe rate, the persisting spokes generally contributing to the instability seen at high strobe rates. This issue will be taken up later.

\section{EXPERIMENT 3: \\ Apparent Motion as a Function of Spatial Separation}

There have been many discussions about the perceived velocity of AM. Kolers (1972), for instance, argued that "we do not know at present how best to calculate the per- 


\section{SPOKE WHEEL}

\section{Anticlockwise Rotation}

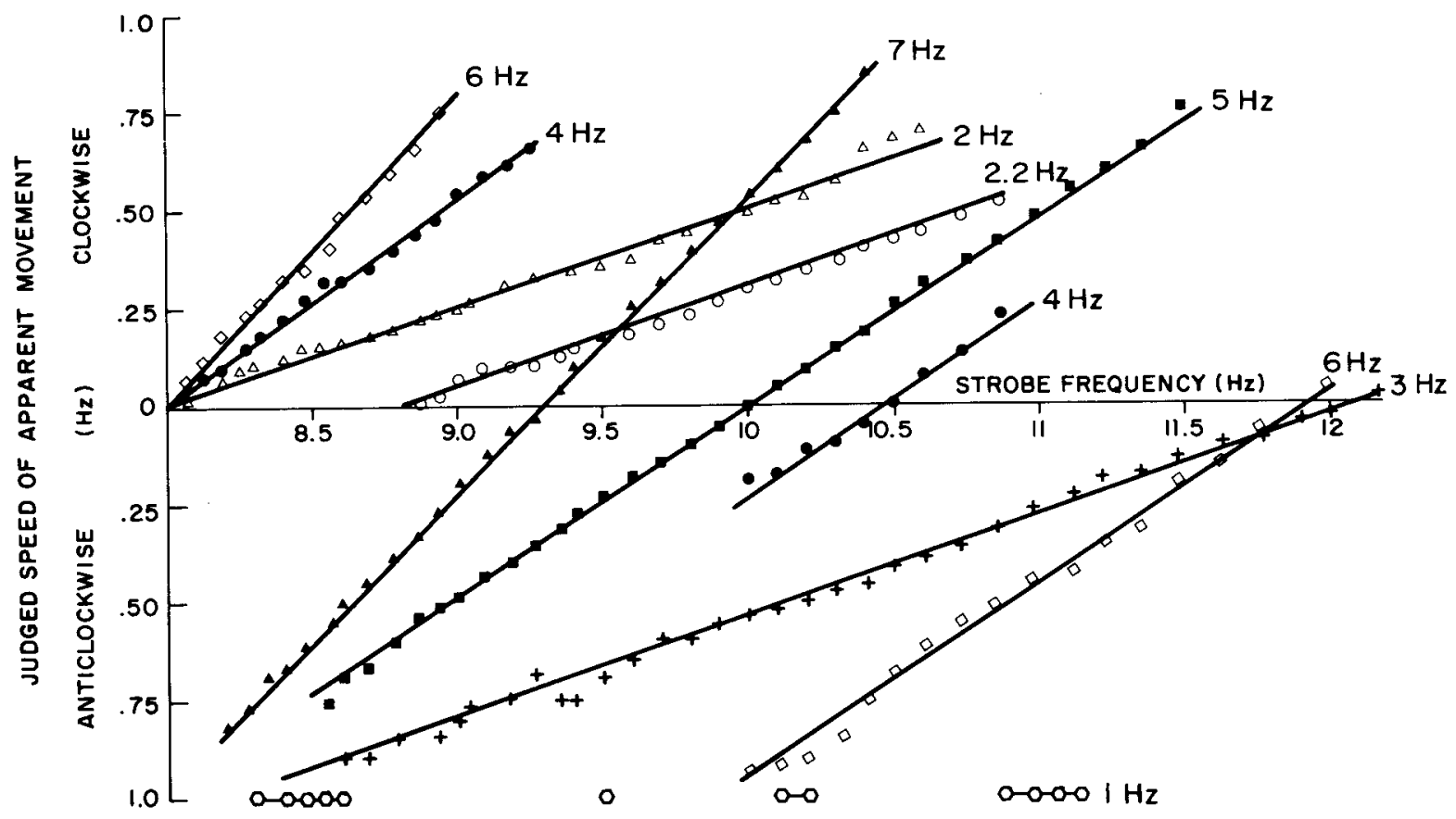

Figure 2. Averaged judged rotation speed as a function of strobe frequency. For all judgments of speed, on duration was 1 msec. The parameter appearing beside each curve is the rotation rate of the wheel.

ceived velocity of the illusory object"' (p. 19) because, among other things, it was not clear what the appropriate temporal parameter should be. Experiments 1 and 2 show that SOA provides an adequate parameter for the WWE and that the range of speeds reported here for AM lies comfortably within the range over which RM is seen. Two questions remain: Can we find a simple and appropriate spatial parameter to describe the WWE motion effects? How closely is the WWE related to the beta motion clasically defined as object AM between collections of two or more discrete displays?

Experiment 3 examined the possibility that the appropriate spatial variable is the angular separation between an illuminated spoke and its position at the next flash. That is, for a given constant rotation rate and a given constant strobe rate, successive exposures will illuminate the spokes either in exactly the positions previously occupied by spokes (exact repetition) or in positions at fixed angular separations from the positions occupied by spokes at the onset of the previous flash (Experiment 1). This may seem to be too obvious a matter to be worth investigating, but it should be noted that defining "nearest neighbor" in terms of spoke position implies a definite hypothesis about the way in which the display is perceived. At the $5-\mathrm{Hz}$ rotation rate used here, a $1-\mathrm{msec}$ increase or decrease in SOA changes the angular separation between spokes by $1.8^{\circ}$. The actual separation between "corresponding" points on the two spokes varies systematically, of course, as a function of their distance from the center of rotation.

\section{Procedure}

The four-spoke wheel was rotated in a counterclockwise direction at $5 \mathrm{~Hz}$ and the strobe frequencies were varied in 1-msec steps around the center frequencies, 80, 40, 20, 10, 5, 4, 3.33, 2.5, and $2 \mathrm{~Hz}$, all of which provide exact repetitions of the stimulus pattern. The exact repetitions at these strobe rates require some explanation. Obviously, any progression through $90^{\circ}$, or a multiple of $90^{\circ}$, will result in exact repetition for a four-spoke wheel. This angular displacement occurred at a strobe rate of $20 \mathrm{~Hz}$, whereas the strobe rates of $10,5,4,3.3,2.5$, and $2 \mathrm{~Hz}$ produced multiples of $90^{\circ}-2,4,5,6,8$, and 10 , respectively. For the $40-$ and $80-\mathrm{Hz}$ strobe rates the angular displacements for each strobe were $45^{\circ}$ and $22.5^{\circ}$. Displacements of this size for lower strobe rates would naturally produce AM, but this does not occur here, presumably because of persistence (see below). The speed and direction of the AM were measured as before for values on either side of the nine center frequencies above, that is, by incrementing and decrementing the SOAs for the center frequencies in 1-msec steps.

\section{Results}

In Figure 3, speed and direction of motion are plotted against displacement angle away from exact repetition, with the strobe center frequency as the parameter. Again, it is clear that the data are very systematic and indicate that increases in strobe frequency result in faster and faster AM for a given angular displacement. In fact, the speed of AM can be predicted almost exactly if the angular displacement and the appropriate SOA value are known: speed of $A M$ rotation = angular displacement $/$ SOA. For example, consider the angular displacement of $-16.2^{\circ}-$ that is, an angular displacement of $16.2^{\circ}$ away from exact repetition, leading to clockwise AM. For the $10-\mathrm{Hz}$ 


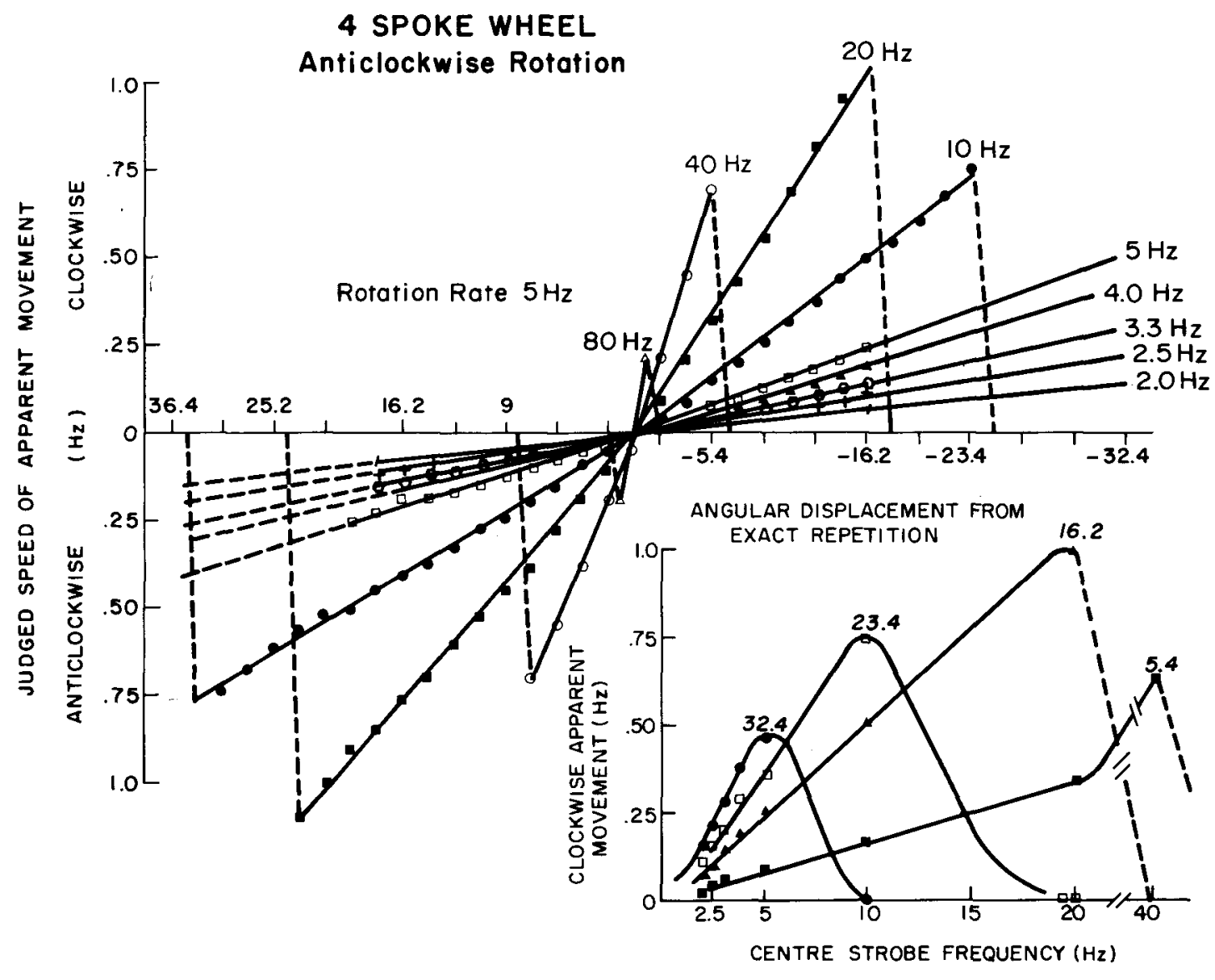

Figure 3. Averaged judged rotation speed as a function of angular displacement from exact pattern repetition for nine center strobe frequencies. The strobe duration was 1 msec. The insert with data extracted from the main figure shows clockwise apparent movement speed as a function of the center strobe frequency for four angular displacements: $32.4^{\circ}, 23.4^{\circ}, 16.2^{\circ}$, and $5.4^{\circ}$.

center strobe frequency curve, exact repetition $\left(0^{\circ}\right)$ occurs for an SOA of $100 \mathrm{msec}$ and the $16.2^{\circ}$ displacement with a 9-msec decrement in this SOA (i.e., $9 \times 1.8^{\circ}=$ $16.2^{\circ}$, the SOA being $\left.91 \mathrm{msec}\right)$. The predicted value of perceived rotation will therefore be $0.49 \mathrm{~Hz}$ [ $(16.2 \times$ $1000) /(360 \times 91)]$, compared with the measured value of $0.5 \mathrm{~Hz}$. Similar calculations can be made for any point on the figure. The correlation between the measured values of apparent rotation speed and calculated or predicted speed for the data points in Figure 3 is 0.99 .

Certain complications arise at higher strobe rates because of visual persistence (see Finlay et al., 1984). For example, at $40 \mathrm{~Hz}$, the 4-spoke wheel is stationary and appears to have 8 spokes. Exact repetition will occur, then, if the wheel traverses $45^{\circ}$. Similarly, at $80 \mathrm{~Hz}$, the 4-spoke wheel appears to have 16 spokes, and exact repetition therefore will occur with displacements of $22.5^{\circ}$. Displacements away from exact repetition can be displacements from immediately preceding strobe flashes or from flashes two or three strobes earlier. It is important to note that when these persisting spokes move, they do so with the same apparent velocity as other spokes. In fact, it is not possible to discern which are spokes resulting from more recent strobe flashes.
The insert in Figure 3 is an attempt to display the data in a form more directly comparable to the usual method of depicting beta motion. The data graphed are speeds of clockwise apparent motion against center strobe frequency, the parameter being angular displacement away from exact repetition. For large angular displacements, the center strobe frequency range over which AM occurs is quite narrow, and with decreasing angular displacement both the strobe frequency range and the apparent speed measured increase.

\section{DISCUSSION}

It is clear that apparent speed of the WWE in the conditions of these experiments is determined by SOA and the angular displacement of successively illuminated spokes away from exact repetition. Such a simple conclusion has never been possible with the classical beta motion displays. There are certain differences between the AM of the WWE and the beta motion of the traditional demonstrations. In the first place, we note that our calculations of predicted speed involve angular displacements on the wheel, but not visual angle subtended at the eye. It is well documented that AM in two-point displays is 
very dependent upon visual angle (Neuhaus, 1930), but doubling the viewing distance to the wheel, and thus varying visual angle substantially, has no effect on the speed of apparent rotation. In other respects, too, the WWE is incredibly robust; it is not affected, within obvious limits, by the ambient illumination or the strobe intensity. It is also unaffected by contrast reversal (black spokes on a white wheel). The WWE thus displays many of the constancy properties associated with physical objects and real motion.

Differences between the WWE and beta motion can be summarized as follows. (1) The WWE is seen over a wide range of temporal frequencies, whereas the temporal frequency range of visual beta motion is quite limited (Caelli \& Finlay, 1979; Finlay \& Caelli, 1979). (2) As pointed out above, visual angle subtended is a critical variable in beta motion, but, at least for the limits used here, it is not critical for the WWE. (3) The occurrence of beta motion varies with stimulus duration, but for the WWE stimulus conditions of these experiments, duration is not at all critical. Given these differences, is it reasonable to suppose that the WWE is just another manifestation of beta motion, as Wertheimer (1912/1961) suggested? In fact, although many alternative displays that give rise to AM have been described, we lack an effective taxonomy for AM or a consistent set of experimental paradigms that define it. It is clear that factors of figural organization play an important role in many cases (e.g., Caelli \& Dodwell, 1980; Kolers, 1972), but the nature of the perceptual organization involved has not always been clearly identified. Ullman (1979) proposed that the correspondence process in AM is "low level and autonomous and that it establishes matches between elementary constituents of images on the basis of built in affinity measures and local interactions" (p. 8). This proposal is too vague to be useful, unless its terms are defined. Nevertheless, our findings are consistent with it, to the extent that they are explicable in terms of SOA and angular displacement. This implies that spokes are image primitives, and that angular separation is the appropriate nearest-neighbor (affinity) measure. Thus a particular perceptual organization leads to a specific form of AM, and the question to ask is whether it is sui generis or is to be subserved under a more general category such as beta motion.

In view of the number of differences we have identified between the WWE and beta motion, it is tempting to claim that they are separate phenomena. On the other hand, the rate of spatiotemporal sampling, or the amount of interpolation leading to the perception of motion, is very different in the two cases. It is possible to obtain a rotational component in AM between two nonparallel lines, which seems to have classical beta motion characteristics. A wide range of AM with different rotational components is possible right up to the full WWE, but the properties of these displays have not been explored. It is not known whether the robustness, constancy properties, and ease of measurement of the WWE are mainly due to the perceptual organization per se (a spoked wheel rotating), to overdetermination through high sampling rates that may approximate the stimulating conditions of real motion, or both.

Contrary to some claims (e.g., Kaufman et al., 1971), the range of speeds over which the WWE can be measured overlaps the range of speeds for the perception of RM. Perhaps more interestingly, there is no interaction under the conditions of these experiments between the RM of the wheel and the speed of AM reported. That is, there is no biasing of the WWE's AM in the direction of the actual rotation of the wheel. This was unexpected, as several studies have shown effects of RM on the perception of AM (Clatworthy \& Frisby, 1973; Finlay et al., 1984; Green \& von Grünau, 1983). Our results do not speak directly to the issue of the relationship between RM and $A M$, but they do suggest that the possible differences in processing mechanisms for translational and rotational components of both forms of motion are worth further exploration.

\section{REFERENCES}

ATtNeAve, F., \& Block, G. (1973). Apparent movement in tridimensional space. Perception \& Psychophysics, 13, 301-307.

Bartley, S. H. (1941). Vision. New York: Van Nostrand.

Caell, T. M., \& Dodwell, P. C. (1980). On the contours of apparent motion: A new perspective on visual space-time. Biological Cybernetics, 39, 27-35.

Caelli, T. M., \& Finlay, D. (1979). Frequency, phase, and colour coding in apparent motion. Perception, 8, 59-68.

Clatworthy, J. L., Frisby, J. P. (1973). Real and apparent movement: Evidence for a unitary mechanism. Perception, 2, 161-164.

Finlay, D. (1982). Stroboscopic motion in depth. Perception, 11, 733-741.

Finlay, D., \& Caelli, T. M. (1979). Frequency, phase, and colour coding in apparent motion: 2. Perception, 8, 595-602.

Finlay, D., Dodwell, P. C., \& Caelu, T. M. (1984). The waggonwheel effect. Perception, 13, 237-248.

Foster, D. H. (1975). Visual apparent motion and some preferred paths in the rotation group SO(3). Biological Cybernetics, 18, 81-89.

GreEN, M., \& von GrünAU, M. (1983). Real and apparent motion: One mechanism or two? ACM Siggraph/Sigart Newsletter, 17, 17-22.

Kaufman, L., Crrulnik, I., Kaplowitz, J., Melnick, G., \& STOFF, D. (1971). The complementarity of apparent and real motion. Psychologische Forschung, 34, 343-348.

Kolers, P. A. (1972). Aspects of motion perception. New York: Pergamon Press.

Neuhaus, W. (1930). Experimentelle Untersuchungen der Scheinbewegung. Archiv für Gesamte Psychologie, 75, 315-458.

Rock, I., \& Ebenholtz, S. (1962). Stroboscopic movement based on change of phenomenal location rather than relative location. American Journal of Psychology, 75, 193-207.

Ullman, S. (1979). The interpretation of visual motion. Cambridge, MA: MIT Press.

WERTHEIMER, M. (1961). Experimentelle studien uber das sehen von Bewegung. In T. Shipley (Ed.), Classics in psychology (pp. 10321089). New York: Philosophical Library. (Reprinted from Zeitschrift für Psychologie, 1912, 61, 161-265)

(Manuscript received August 24, 1984; revision accepted for publication September 25, 1986.) 